\title{
Drifting Plasma Collection by a Positive Biased Tether Wire in LEO-Like Plasma Conditions: Current Measurement and Plasma Diagnostic
}

\author{
Jean-Michel Siguier, Pierre Sarrailh, Jean-François Roussel, Virginie Inguimbert, Gaël Murat, and Juan SanMartin
}

\begin{abstract}
BETs is a three-year project financed by the Space Program of the European Commission, aimed at developing an efficient deorbit system that could be carried on board any future satellite launched into Low Earth Orbit (LEO). The operational system involves a conductive tape-tether left bare to establish anodic contact with the ambient plasma as a giant Langmuir probe. As a part of this project, we are carrying out both numerical and experimental approaches to estimate the collected current by the positive part of the tether. This paper deals with experimental measurements performed in the IONospheric Atmosphere Simulator (JONAS) plasma chamber of the OneraSpace Environment Department. The JONAS facility is a $9-\mathrm{m}^{3}$ vacuum chamber equipped with a plasma source providing drifting plasma simulating LEO conditions in terms of density and temperature. A thin metallic cylinder, simulating the tether, is set inside the chamber and polarized up to $1000 \mathrm{~V}$. The Earth's magnetic field is neutralized inside the chamber. In a first time, tether collected current versus tether polarization is measured for different plasma source energies and densities. In complement, several types of Langmuir probes are used at the same location to allow the extraction of both ion densities and electron parameters by computer modeling (classical Langmuir probe characteristics are not accurate enough in the present situation). These two measurements permit estimation of the discrepancies between the theoretical collection laws, orbital motion limited law in particular, and the experimental data in LEO-like conditions without magnetic fields. In a second time, the spatial variations and the time evolutions of the plasma properties around the tether are investigated. Spherical and emissive Langmuir probes are also used for a more extensive characterization of the plasma in space and time dependent analysis. Results show the ion depletion because of the wake effect and the accumulation of ions upstream of the tether. In some regimes (at large positive potential), oscillations are observed on the tether collected current and on Langmuir probe collected current in specific sites.
\end{abstract}

Index Terms-Ionospheric plasma, spacecraft charging, tether satellite.

\section{INTRODUCTION}

$\mathbf{T}$ HE objective of this paper is to better understand the current collection on a bare tether to provide an estima- tion of the current carried along by the tether. The orbital tether dynamic creates an electromotive force along the tether of $\sim 100 \mathrm{~V} / \mathrm{km}$ [1]. Thus, the electric potential along the tether goes from highly positive potential to highly negative potential compared with the Low Earth Orbit (LEO) particle energy.

As a low ionic current is collected by the negative biased part of the tether, this section is not sufficient to be the single cathode of the tether. Thus, most of the time, a more efficient cathode system is added to the tether to insure the current loop in the tether [2] and [3]. The most interesting part of the tether is the positive biased part of the tether. Because of the highly positive potential reached (in comparison with the electron temperature), high electronic current is collected. The orbital velocity of the tether makes ions having a drifting velocity in the tether reference. This velocity is not sufficient to make the ions reach the tether. In the most efficient part of the tether, the directed energy of ions is lower than the tether potential hence all the ions are repelled. Nevertheless, it is well known that the orbital motion limited (OML) like theory provides a good estimation for nonflowing plasma but discards for flowing plasma encountered in this case [4]. An evaluation of the current collection is also essential because it constitutes an input to determine the thrust of the tether [5].

However, the scientific literature concerning modeling [6]-[8] and measurements [9]-[11] of current collection by a positive biased wire in a mesothermal LEO plasma flow is inconclusive up to now.

As a support to ongoing modeling works [12], we performed several types of measurements:

1) tether collected currents versus tether polarization with several plasma source conditions;

2) spatial variations and time evolutions of the plasma properties around the tether.

\section{EXPERIMENTAL SETUP}

The JONAS facility is a $9-\mathrm{m}^{3}$ vacuum chamber equipped with two $10-\mathrm{keV}$ electron guns, a plasma source and several electrostatic discharges (ESDs), and plasma diagnostic devices such as transient current probes, surface potential probes, Langmuir probes, and so on.

A cryogenic pump gives a working pressure $<10^{-7} \mathrm{hPa}$. The working pressure with plasma is $\sim 5 \times 10^{-6} \mathrm{hPa}$. In this condition, ionization in the sheath can be neglected [11].

The plasma source is a Kauffman type, providing 10 to $30-\mathrm{eV}$ drifting $\mathrm{Ar}^{+}$ions. The energy range corresponds 


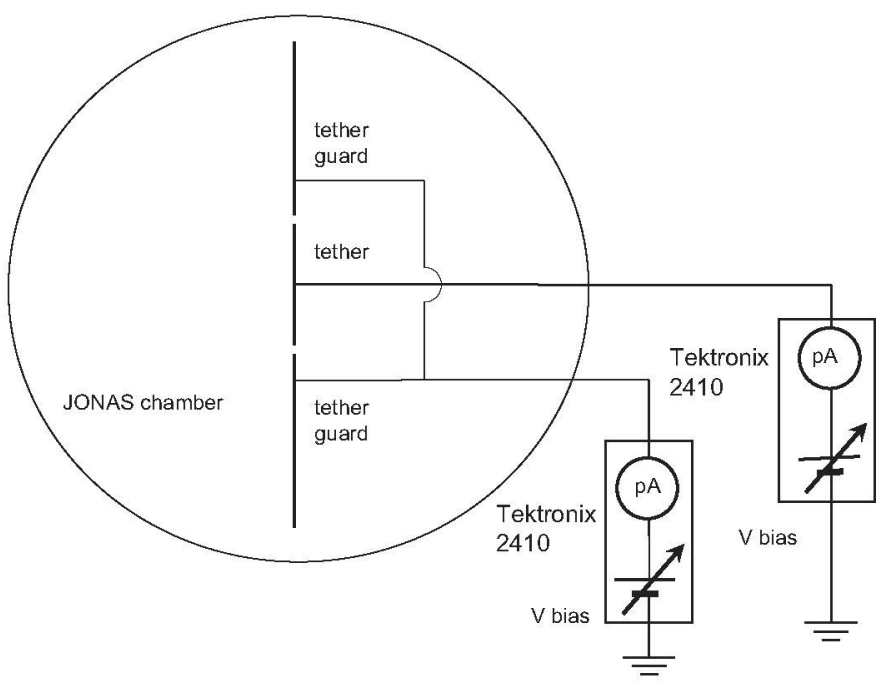

Fig. 1. Experimental setup.

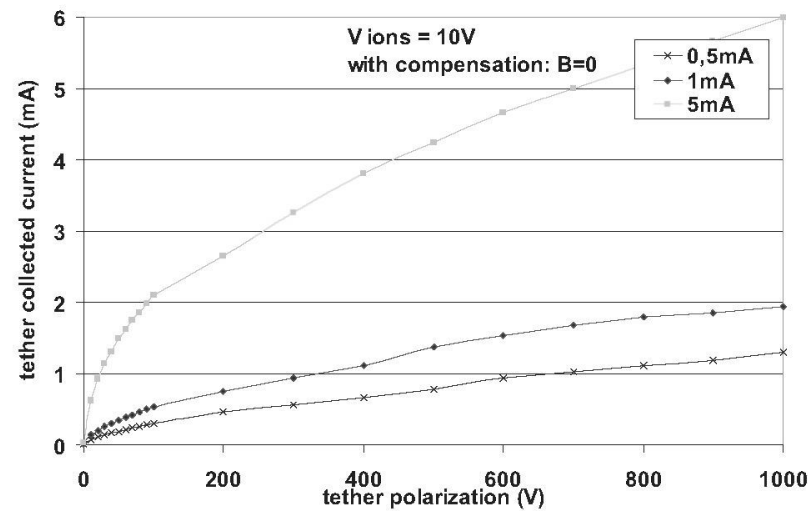

Fig. 2. Tether collected current with $10-\mathrm{V}$ ion acceleration and different plasma ion currents.

to the orbital velocity $(8 \mathrm{~km} / \mathrm{s}$ for $20 \mathrm{~V})$. This plasma is constituted of two ions populations: drifting ions and low energy ions (after interaction with neutrals). Rough characteristics are: $\mathrm{N}_{i}=10^{11}$ to $10^{12} \mathrm{~m}^{-3}, \mathrm{~T}_{e}\left(\approx \mathrm{T}_{\text {ions }} \approx \mathrm{T}_{\text {neutrals }}\right)=$ 0.1 to $0.2 \mathrm{eV}$. Because the purpose of this paper is to determine the physical phenomena, we use argon, which is the inert gas most similar to oxygen (LEO plasma constituent). We thought that phenomena will be the same for different gas but with different energies, that is different applied potentials.

Earth magnetic field is compensated by external coils within a volume of $\sim 1 \mathrm{~m}^{3}$ in the centre of the chamber. All experimental results are produced in the earth's magnetic field compensated volume.

Plasma diagnostics are achieved using different types of Langmuir probes (spherical, guarded plane, triple, and emissive probes) set in a 2-D displacement system.

The tether is a metallic wire (5-mm diameter) fixed vertically in the middle of the JONAS chamber (Fig. 1), that is perpendicularly to the plasma drift. To avoid ends effects [9], it constitutes three parts:

1) central part (length $=30 \mathrm{~cm}$ ) positively biased where the collected current is measured;

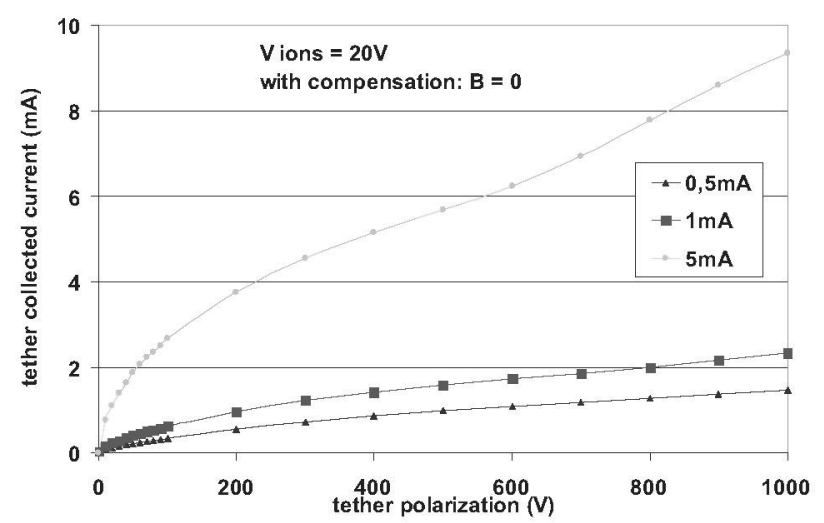

Fig. 3. Tether collected current with $20-\mathrm{V}$ ion acceleration and different plasma ion currents.

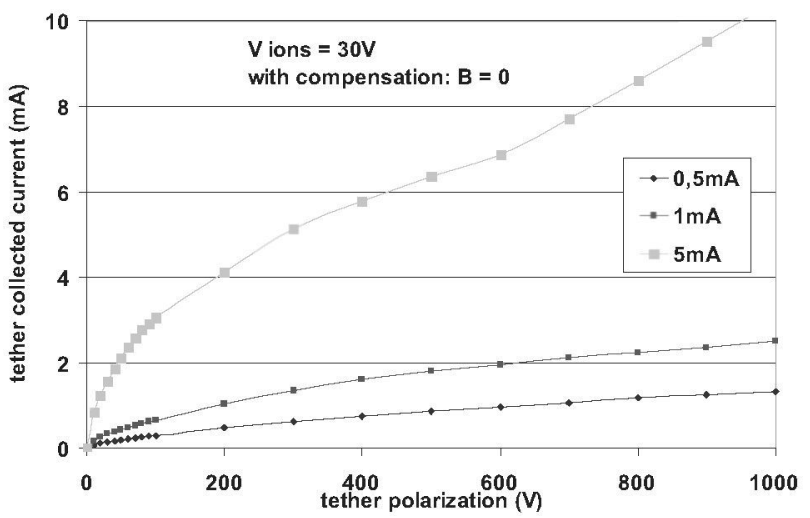

Fig. 4. Tether collected current with 30-V ion acceleration and different plasma ion currents.

2) upper and a lower parts (length $=30 \mathrm{~cm}$ each) biased to the same voltage as the central part but no current is measured. They are acting as guards simulating an infinite tether length for the central part.

\section{Plasma Collected Currents With a Positive BIASED TETHER WIRE}

\section{A. Experimental Results}

The aim of this first setup is to measure the collected currents of a positively biased tether wire in several drifting plasma densities and velocities simulating ionospheric conditions.

Plasma source varies from 10 to $30 \mathrm{~V}$ for ion energy and 1 to $5 \mathrm{~mA}$ for ion current. The ion flux is neutralized by an electron source set after the acceleration grid by a heated tungsten filament, this system insuring the lack of high energy electrons in the vacuum chamber. For each plasma source values, tether polarizations vary from 0 to $+1000 \mathrm{~V}$. For all measurements, geomagnetic field is neutralized by external coils. Current/voltage characteristics are shown in Figs. 2-4.

\section{B. Discussion}

In Figs. 2-4 as in the OLM theory, the collected current increases as a function of the tether potential. In Fig. 5, the discrepancy compared with the 2-D OML theory is plotted. 


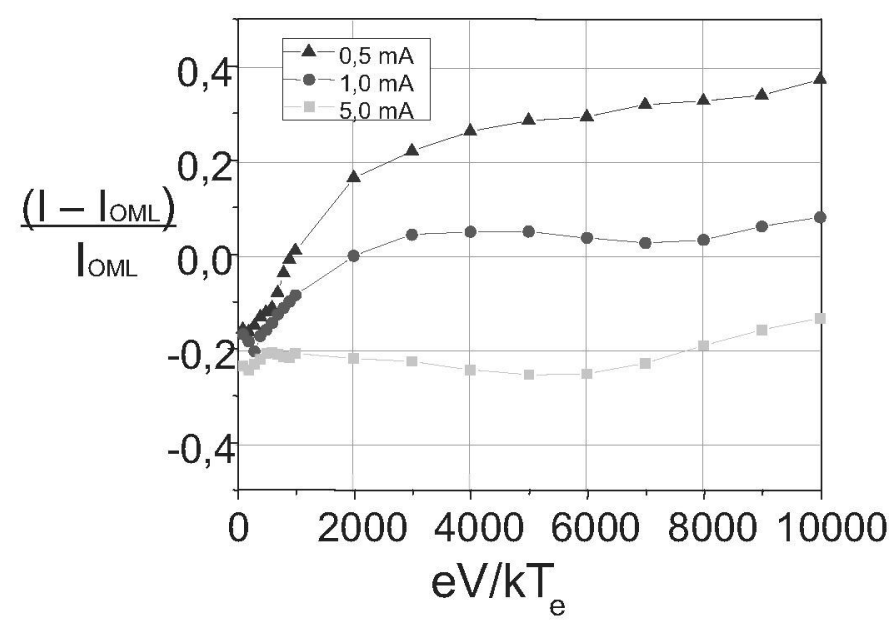

Fig. 5. Collected current discrepancy in comparison to OML theory as a function of reduced potential.

To calculate the OML current, the value of plasma density and electron temperature is taken from plasma probe measurement centered on the tether position but without the tether. The temperature value is considered to be $0.1 \mathrm{eV}$. The plasma densities are $1.2 \times 10^{11}, 2.4 \times 10^{11}$, and $1.2 \times 10^{12} \mathrm{~m}^{-3}$ for plasma source currents of, respectively, 0.5, 1.0, and 5.0 mA. We can notice that the best agreement between the OML theory and the current measurement is obtained for the case $1.0 \mathrm{~mA}$. In fact, the plasma density is measured accurately for our standard source conditions ( $20 \mathrm{~V}-1 \mathrm{~mA}$ ). Other source conditions are less investigated and thus plasma density is less accurate. More generally, we found a good agreement between the OML theory and the measurement, i.e., $+/-30 \%$, in most of the tested regimes. We can also observe that the deviation is independent of the potential for $5.0 \mathrm{~mA}$ but it is not the case for the lower currents. This is probably because of the fact that the sheath size is proportional to the Debye length. When the plasma density is lower, the sheath radius is larger and this can have three kinds of effects:

1) the sheath reaches the chamber walls. In these zones, the plasma is completely depleted and cannot contribute to the current collection. In consequence, the collected current decreases. As the measured collected current increases, current variations are not because of this phenomenon;

2) the sheath radius increases and reaches the zones where the plasma density is higher. In the JONAS chamber, we can see in the next section that the plasma density decreases as $1 / \mathrm{r}^{2}$ from the plasma source;

3 ) the sheath radius becomes in the same order of magnitude as the sample total length $(1 \mathrm{~m})$. In this case ends effects produce an increase of the current collection.

\section{Plasma Mapping Around the Tether With a Spherical Langmuir Probe Biased at $-5 \mathrm{~V}$}

\section{A. Experimental Results}

The following measurements are performed for plasma characterization around the tether wire positively biased. The plasma source configuration is $20 \mathrm{~V}-1 \mathrm{~mA}$.

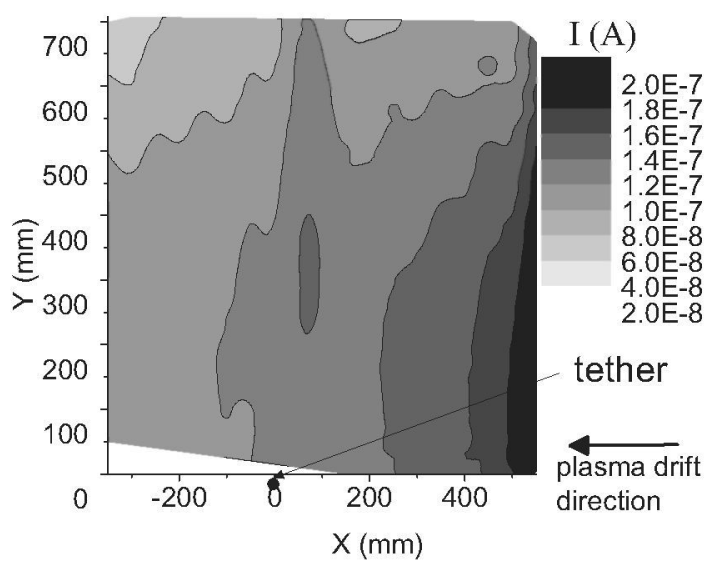

Fig. 6. Plasma mapping with Langmuir probe collected current at $-5 \mathrm{~V}$ for a tether polarization of $0 \mathrm{~V}$.

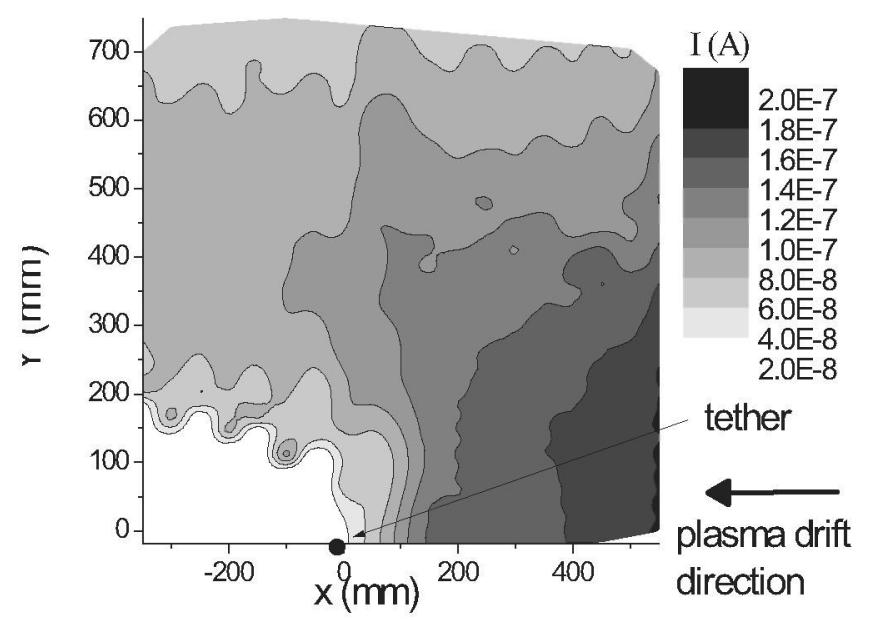

Fig. 7. Plasma mapping with Langmuir probe collected current at $-5 \mathrm{~V}$ for a tether polarization of $+100 \mathrm{~V}$.

The tether is biased at different potential from zero to $+1000 \mathrm{~V}$ and a spherical Langmuir probe is moved around it in a plane perpendicular to the tether axis.

Because the drifting plasma is highly perturbed by tether biasing, I(V) probe characteristics are not exploitable for plasma parameters determination. Thereby, only ions collected current, with a negatively biased probe, for different tether polarizations are shown in Figs. 6-8. The probe is $2-\mathrm{mm}$ diameter and is biased at $-5 \mathrm{~V}$. This selected potential is significantly lower than ions energy $(20 \mathrm{eV})$. Thus, we can suppose that the plasma surrounding the probe is not significantly disturbed by the probe. In this conditions, the spatial variation of the probe collected current permits to have a good estimation of the plasma density variation in the chamber. In these figures, tether is perpendicular to $(\mathrm{X}, \mathrm{Y})$ plane and located at $\mathrm{X}=0$ and $\mathrm{Y}=0$. Plasma is drifting from the positive $\mathrm{X}$ direction toward the negative $\mathrm{X}$ direction. The spherical Langmuir probe is moved in the $(\mathrm{X}, \mathrm{Y})$ plane and, for symmetrical reasons, only half of the plane is scanned. 


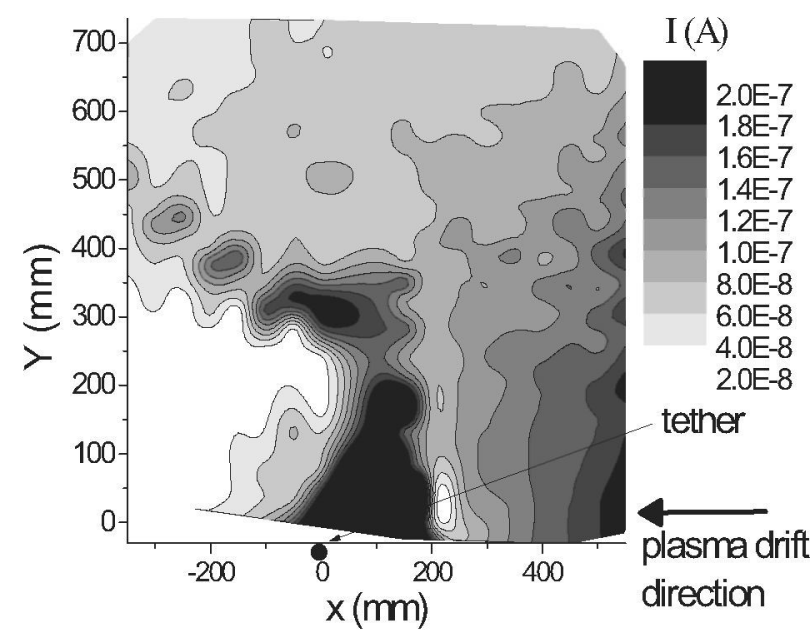

Fig. 8. Plasma mapping with Langmuir probe collected current at $-5 \mathrm{~V}$ for a tether polarization of $+400 \mathrm{~V}$.

\section{B. Discussion}

For $0-\mathrm{V}$ tether biasing (Fig. 6), no plasma disturbance is observed. As expected, the collected ion current increases when the probe is getting closer to the source in a $1 / \mathrm{r}^{2}$ law.

From $+100-\mathrm{V}$ tether biasing and more (Fig. 7), we can see an ions depletion zone around the tether. In this zone, the current collected by the probe rapidly fall down to zero. This zone corresponds to a wake formation. Because of the probe potential is well higher than the ion drifting energy $(20 \mathrm{eV})$, ions coming from the source are deviated by the tether sheath in direction of the chamber walls.

For higher potentials (Fig. 8), the probe current increases in the ram direction and by the side of the wake corresponding to an increase of plasma density because of ions repulsion in the tether sheath. As a remark, peaks of current correspond to measurements points and the decreases of currents between peaks are artifact because of the plotter interpolation. In reality, the over density zone is continuous between peaks. This phenomenon tends to increase with the potential because of the fact that the sheath radius increases with the tether potential.

Above $600 \mathrm{~V}$, the plasma is disturbed in the entire chamber.

\section{Plasma Mapping Around the Tether With an EMISSIVE LANGMUIR PROBE}

\section{A. Experimental Results}

To measure local potential around the tether, we implanted an emissive probe device. This probe is a heated and polarized tungsten filament. A potential sweep applied to this filament gives the plasma potential [13].

Local potential is measured in two directions around the tether positively biased at $+400 \mathrm{~V}$. The emissive probe is moved along the plasma source/tether axis and perpendicularly to this axis. Plasma source conditions are: $20 \mathrm{~V}-1 \mathrm{~mA}$.

Fig. 9 shows the local potential when the emissive probe moves from the tether to the plasma source (ram direction). It shows a strong decrease of the local potential from the tether to a distance $\sim 200 \mathrm{~mm}$ where the local potential reaches the plasma potential. This decrease corresponds to

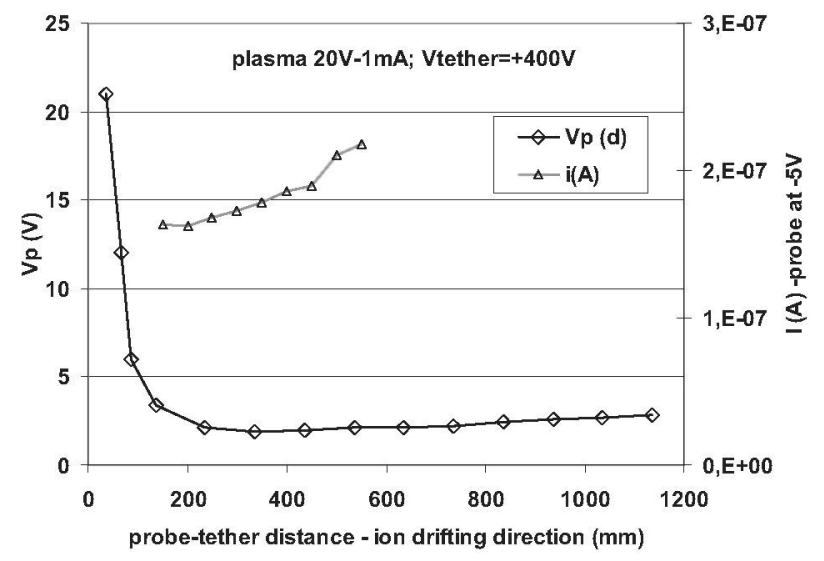

Fig. 9. Local potential and spherical probe current function of the ther (distance $=0$ ) in the ram direction.

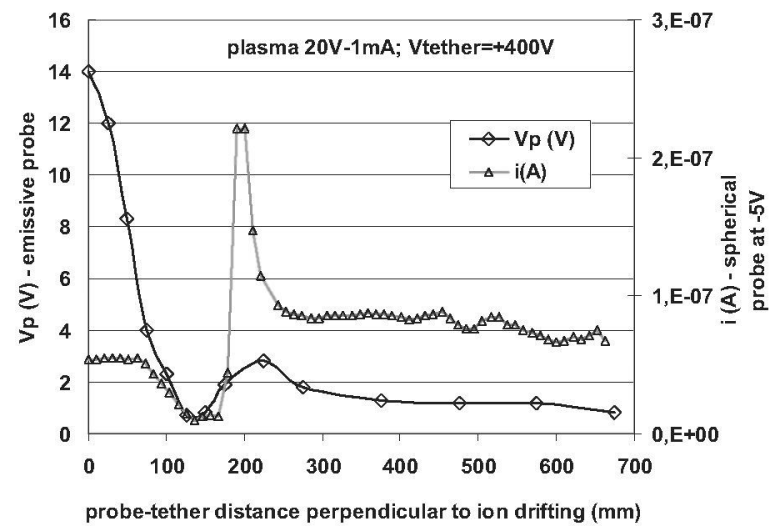

Fig. 10. Local potential and spherical probe current function of tether distance perpendicularly to the ram direction from $5 \mathrm{~cm}$ behind the tether (relatively to the ram direction) toward the chamber wall.

the sheath zone. The tether potential is progressively screened by the surrounding plasma (with a plasma potential of a few Volts in comparison with the ground). When the probe moves away from the tether (the probe is getting closer to the plasma source), plasma potential increases slightly because of the increase of plasma density. Spherical probe current at $-5 \mathrm{~V}$, as measured in the same conditions as in Fig. 8, is shown in this figure. It confirms the increase of plasma density in this direction.

Fig. 10 shows the local potential and spherical probe current when the probes are moved perpendicularly to the plasma source/tether direction, starting from the tether $(5 \mathrm{~cm}$ behind it) toward the chamber wall. As in Fig. 9, we see a strong decrease of the local potential from the tether to a distance $\sim 150 \mathrm{~mm}$ where the local potential reaches the plasma potential value. Then, the local potential increases up to three times the plasma potential at $200 \mathrm{~mm}$ and decreases smoothly to plasma potential value when it get closer to the chamber wall. Spherical probe current at $-5 \mathrm{~V}$, as measured in the same conditions is shown in Fig. 8. Thus, the increase of plasma density $\sim 200 \mathrm{~mm}$ is confirmed by the two types of measurements.

\section{B. Discussion}

Results of Figs. 9 and 10 permit to estimate the sheath length and the position of the over-density zone. 


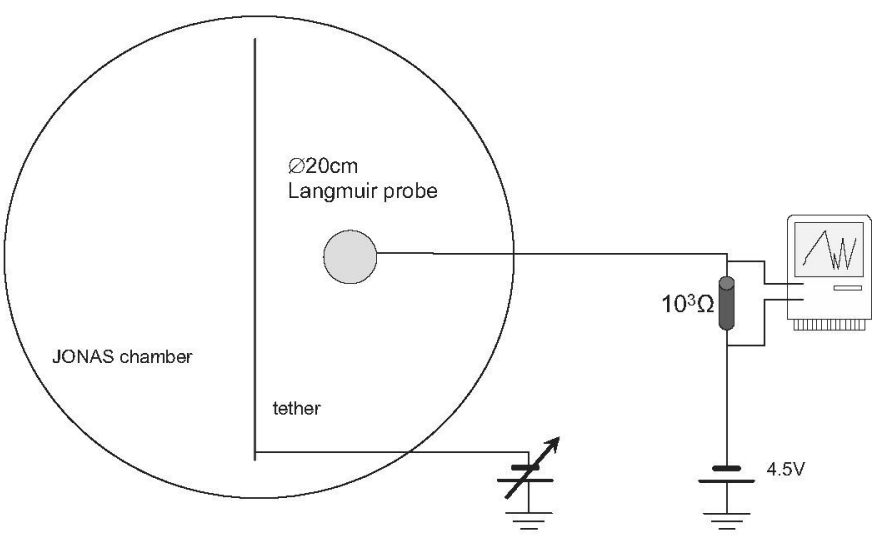

Fig. 11. Plasma oscillations measurement setup with a $20-\mathrm{cm}$ diameter Langmuir probe.

Focusing on Fig. 9, the decrease of the potential is compatible with the standard sheath theories. Considering a thin sheath with a constant density of electrons and no ion (similar to the ions matrix sheath), we measure a sheath length of $300 \mathrm{~mm}$ for a plasma density of $2.4 \times 10^{11} \mathrm{~m}^{-3}$. This value is not so different from emissive probe measurements (the decrease of the potential at the vicinity of the wire because of the cylindrical shape is not considered). From these measurements, the sheath length can be estimated $\sim 150 \mathrm{~mm}$.

The increase of the potential when we get closer to the plasma source is because of the increase of the plasma density as we can see in Fig. 9 (increasing current values). Between the positions at $300 \mathrm{~mm}$ and at $600 \mathrm{~mm}$, the current has increased by a factor of 1.26 and the potential is $0.1-\mathrm{V}$ higher. But, if we consider a Maxwell-Boltzmann evolution of electrons and the quasi-neutrality in the undisturbed plasma, a change in potential of $0.1 \mathrm{~V}$ should increase the density by a factor of 2.7. This discrepancy can also be due to uncertainty on the measurement by the emissive probe or to underestimation of the electronic temperature.

In Fig. 10, the value of the sheath width is about the same as in the ram direction. This tends to prove that the sheath shape is quite cylindrical in the ram side. The most interesting point is to observe the effect of the ions deviation by the sheath. This deviation creates a wake but also makes an overdensity zone in the vicinity of the wake. As a consequence of this deviation, the potential in the wake tends to be negative (electron density is greater than ion density). A potential increase is also observed at $200 \mathrm{~mm}$ that is consistent with the ion density increase (i.e., probe ion current measurement). In this situation, the ion density is well higher than the plasma density anywhere else in the chamber. Following Laframboise theory [4], in a static and collision free situation, electrons density never exceeds the density at infinity. Measurement of the potential shows that, in this zone, the potential increases by $2 \mathrm{~V}$, considering an electron temperature of $\sim 0.1 \mathrm{eV}$, in an undisturbed plasma, electrons gain 20 times their energy at this potential peak. In this condition, the neutralization by the ambient plasma is not possible. The ion density is not compensated by the electrons. This kind of neutralization problem frequently creates instabilities in the plasma.

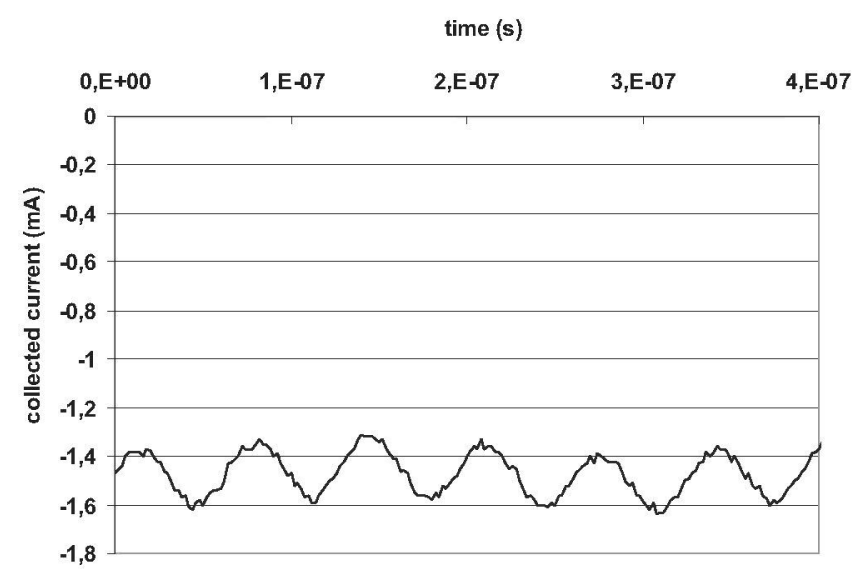

Fig. 12. Oscillations of Langmuir probe collected current at $10 \mathrm{~cm}$ of the tether polarized at $+260 \mathrm{~V}$.

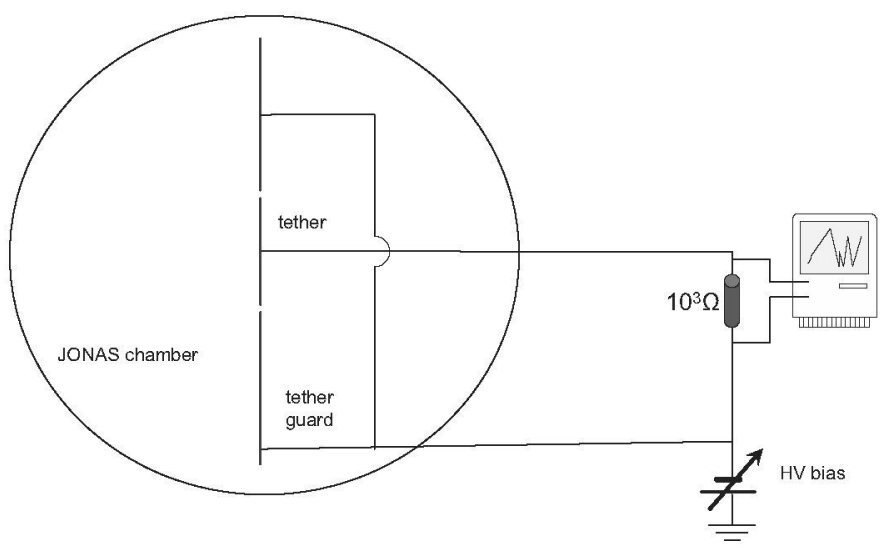

Fig. 13. Plasma oscillations measurement setup with the tether.

The next part is, thus, dedicated to time dependant measurements.

\section{Time Dependance Phenomena: Plasma OsCillations}

\section{A. Experimental Results}

Time-dependant fluctuations of plasma are studied with two different methods.

The first one uses a $20-\mathrm{cm}$ diameter Langmuir probe to have a large collected current and hence sufficient sensitivity to detect small phenomena. The setup is shown in Fig. 11. A $10^{3} \Omega$ resistance is added to the probe polarization circuit and connected to an oscilloscope insulated from the ground.

The probe is positioned at $10 \mathrm{~cm}$ by the side of the tether relatively to the plasma direction.

Fig. 12 shows the total probe current for the following experimental conditions:

1) ions plasma source: $20 \mathrm{~V}-5 \mathrm{~mA}$;

2) tether polarization: $+260 \mathrm{~V}$.

Oscillations, of amplitude $\sim 7 \%$ of total current, are detected with a tether polarization range from $+250 \mathrm{~V}$ up to $+1000 \mathrm{~V}$ (HV apparatus limit). Period range of $70 \mathrm{~ns}$ is in accordance with electronic plasma frequency for this plasma density (101 ns for $\left.1.2 \times 10^{12} \mathrm{~m}^{-3}\right)$. 


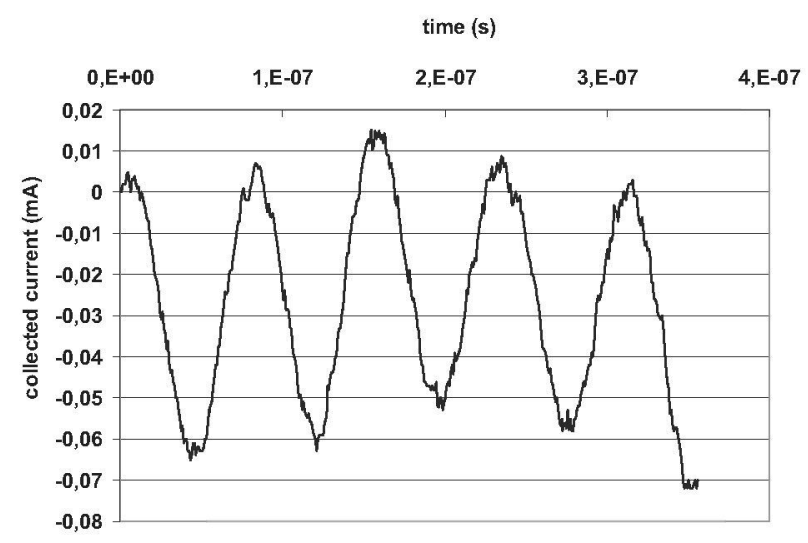

Fig. 14. Fast oscillations of tether collected current polarized at $110 \mathrm{~V}$.

Oscillations period decreases slightly (few \%) when plasma source current is increased from 1 to $5 \mathrm{~mA}$.

In the second method, we measure oscillations of tether collected current. Setup is shown in Fig. 13. Because oscillations are small compared with the constant tether collected current $[2.7 \mathrm{~mA}$ at $+110 \mathrm{~V}]$, this current is filtered by the scope hence only oscillations are detected.

We found two frequency range oscillations.

The shortest period (Fig. 14) is $\sim 80$ ns corresponding to the same one detected with the spherical Langmuir probe for the following experimental conditions:

1) ions plasma source: $20 \mathrm{~V}-4.5 \mathrm{~mA}$;

2) tether polarization: $+110 \mathrm{~V}$;

3) total tether current $=2.7 \mathrm{~mA}$.

These fast oscillations occur with a tether polarization from $+60 \mathrm{~V}$ up to $+300 \mathrm{~V}$. Amplitude is $1.3 \%$ of total tether current.

The longest period (Fig. 15) is $\sim 8 \mu$ s. Experimental conditions are:

1) ions plasma source: $20 \mathrm{~V}-2.5 \mathrm{~mA}$;

2) tether polarization: $+210 \mathrm{~V}$;

3) total tether current $=2.7 \mathrm{~mA}$.

They appear with a tether polarization from $+100 \mathrm{~V}$ up to $+500 \mathrm{~V}$. Amplitude is $0.6 \%$ of total current. They are attributed to the drifting ions.

\section{B. Discussion}

Fast oscillations corresponding to the electronic plasma frequency are visible on the tether collected current and on the Langmuir probe in the plasma. The origin of these oscillations are thus from the undisturbed plasma. They can be the consequence of the over-density ions zone near the wake. As it is not possible for the electrons to neutralize directly this zone, oscillations of the potential would permit in certain conditions to trap electrons (see [2] about adiabatic trapping and collisional trapping in [8]). These trapped electrons would neutralize the ion density and make the lower potential in this zone. Nevertheless, the current experiments tend to show that this mechanism of electron trapping is not sufficient to completely neutralize this zone. In the current case this phenomena is not sufficiently efficient.

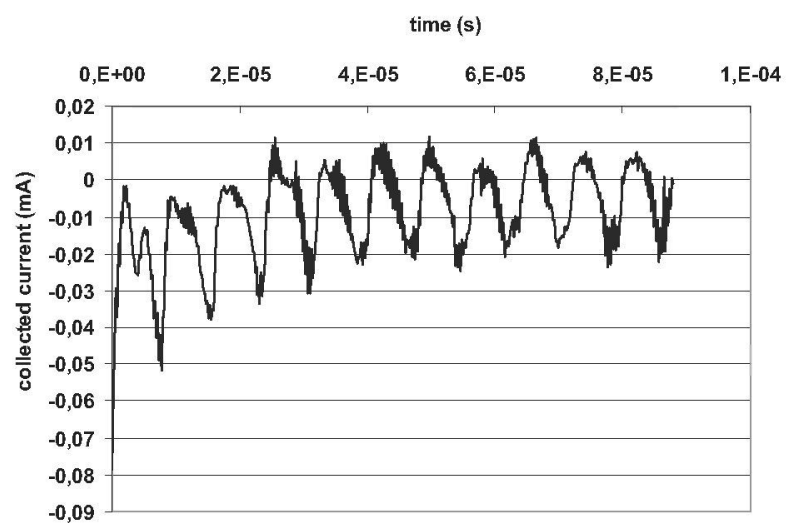

Fig. 15. Slow oscillations of tether collected current polarized at $110 \mathrm{~V}$.

Slow oscillations are also observed. They correspond to ion frequency. The source of these oscillations, only present in the current collected, is not explained. There is no slow oscillation in the current collected by the Langmuir probe.

\section{CONClusion}

We measured the collected currents of a positively biased tether wire in drifting plasma simulating ionospheric conditions. A good agreement with the OML theory was shown in experiments. A deviation was observed depending on the plasma density. This was probably because of the sheath scale in comparison with the wire length and the plasma chamber dimensions.

To verify the accuracy of our measurements, the plasma surrounding tether was studied, showing a wake formation and ions compression zones for specific tether polarizations. A nonneutralized zone was observed where ions were repelled by the sheath. The particularity of this zone was to have an ion density well higher than the maximum electron density (following Laframboise theory). Regarding the limitation of this theory, we excluded the possibility to have collisional effects and we focused on temporal effects.

Time-dependant measurements showed plasma oscillations for specific tether polarizations and plasma conditions. Until now, we can only suppose that oscillations come from the over-density zone. Complementary evidences and in particular modeling effort on this phenomenon will be needed to definitively conclude on it. In this paper, we can also conclude that this phenomenon had a very limited influence on the current collection model.

\section{REFERENCES}

[1] J. R. Sanmartin, M. Charro, X. Chen, E. C. Lorenzini, G. Colombatti, D. Zanutto, J. D. Williams, K. Xie, G. E. Metz, J. A. Carrasco, F. G. De Quiros, O. Kroemer, R. Rosta, T. Van Zoest, J. Lasa, and J. Marcos, "A universal system to de-orbit satellites at end-of-life," J. Space Technol. Sci., to be published.

[2] J. R. Sanmartin, E. C. Lorenzini, and M. Martinez-Sanchez, "Electrodynamic tether applications and constraints," J. Spacecraft Rockets, vol. 47, no. 3, pp. 442-456, 2010.

[3] J. D. Williams and J. R. Beattie, "Spacecraft potential control using plasma contactors," in Proc. 3rd Int. Workshop Interrelationship Between Plasma Experim. Lab. Space, Jul. 1994, pp. 49-65.

[4] J. G. Laframboise and L. W. Parker, "Probe design for orbit-limited current collection," Phys. Fluids, vol. 16, no. 5, pp. 629-636, 1973. 
[5] J. R. Sanmartin, M. Martinez-Sanchez, and E. Ahedo, "Bare wire anodes for electrodynamic tethers," J. Propuls. Power, vol. 9, no. 3, pp. 353-360, May-Jun. 1993.

[6] E. Choiniere and B. E. Gilchrist, "Electron collection to arbitrarily shaped electrodynamic tethers in flowing plasmas: A kinetic model," in Proc. 38th Joint Propuls. Conf., 2002, pp. 1-9.

[7] R. D. Estes, J. Sanmartin, and M. Martinez-Sanchez, "Performance of bare-tether systems under varying magnetic and plasma conditions," J. Spacecraft Rockets, vol. 37, no. 2, pp. 197-204, Mar.-Apr. 2000.

[8] T. Onishi, M. Martinez-Sanchez, and D. L. Cooke, "Effect of magnetic field on current collection to a bare tether in LEO," in Proc. 38th AIAA/ASME/SAE/ASEE Joint Propuls. Conf., 2002, no. AIAA-2002-4051.

[9] B. E. Gilchrist, S. G. Bilén, and A. D. Gallimore, "Current collection to long, thin, probes in dense high-speed flowing plasma," in Proc. Space Technol. Appl. Int. Conf., Sep. 2001, pp. 494-500.

[10] É. Choinière, S. G. Bilén, B. E. Gilchrist, K. R. Fuhrhop, and A. D. Gallimore, "Experimental investigation of electron collection to solid and slotted tape probes in a high-speed flowing plasma," IEEE Trans. Plasma Sci., vol. 33, no. 4, pp. 1310-1323, Apr. 2005.

[11] K. Kashihara, M. Cho, and S. Kawamoto, "Ground experiments and computer simulations of interaction between bare tether and plasma," IEEE Trans. Plasma Sci., vol. 36, no. 5, pp. 2324-2335, Oct. 2008.

[12] P. Sarrailh, J.-F. Roussel, J.-M. Siguier, V. Inguimbert, G. Murat, and J. R. SanMartin, "LEO drifting plasma collection by a positive biased tether wire: Time-dependant simulations using SPIS," in Proc. 12th Spacecraft Charging Technol. Conf., Kitakyushu, Japan, May 2012.

[13] R. Kemp and J. Sellen, "Plasma potential measurements by electron emissive probes," Rev. Sci. Instrum., vol. 37, no. 4, pp. 455-461, Apr. 1966. 